\title{
Clinical and Radiographic Aspects of Metacarpal Remodeling Secondary to a Rubber Band (Latex) Compression in a Dog
}

\author{
Tadeu Basualdo Junior', Joyce Katiuccia Medeiros Ramos Carvalho', João Pedro Brochado Souza², \\ Márcio Virgílio Figueiredo da Silva', Joyce Maira de Araújo' \& Gabriel Utida Eguchi'
}

\begin{abstract}
Background: Musculoskeletal disorders are a common complaint in veterinary small animal casuistic. Along with fractures, degenerative and of carcinogenic etiology are the most frequent and radiographic lesion pattern at these diseases is relative well defined. However, traumatic lesions, considering its innumerous possibilities, may cause unusual clinical and radiographic signs which will delay diagnosis and consequently, adequate treatment. A case of bone osteolysis caused by a compressive trauma by a rubber band is described with its clinical, laboratorial and radiographic aspects.

Case: A 2-year-old female dog was attended at the Veterinary Hospital of the Dom Bosco Catholic University (UCDB), with main complaint being an unresponsive to treatment lesion at the left thoracic limb. At physical examination it was observed lameness of the left thoracic limb with an ulcerative lesion at the palmar surface. At the center of the ulcer a 0.3 $\mathrm{cm}$ line shaped yellow object was identified, similar to a rubber band. Traction was made and the object distended $5 \mathrm{~cm}$ without breaking nor leaving the injury. Showing signs of discomfort, the patient was then sedated for further manipulation. A blood sample for complete blood count and serum biochemistry was collected and radiographic image of the left carpometacarpal-phalangeal region was acquired. Blood analysis revealed moderate thrombocytopenia with an unremarkable serum biochemistry profile (alanine aminotransferase, alkaline phosphatase, creatinine and urea). It was observed metacarpals with increased radiopacity in bone tissue in the mid-diaphysis topography of the II, III, IV and V metacarpal bones, presence of bone remodeling with radiolucent area and slight bone loss (osteolysis) in the mid-diaphysis associated with discrete sclerosis of the medullary cavity of the II, III and V metacarpals. The patient was submitted to surgery and a $3 \mathrm{~cm}$ incision was made following the way of the foreign body, with a small traction the object was removed, confirming the presence of a rubber band. Post-surgery prescription included systemic antibiotic, non-steroidal anti-inflammatory, analgesic and topical ointment. Twenty-two days post-surgery, at revaluation, it was observed only discrete improvement of bone remodeling of $\mathrm{V}$ metacarpal but with complete wound healing and full recovery of the lameness.

Discussion: It was unclear the reason that led to the presence of the rubber band. Unfortunately, the owner could only complaint about at wound that would not heal for weeks. The best hypothesis was the possible use of a beauty accessory after a bathing service. Considering the patient's long hair, detachment of any accessory to a rubber band base could have gone unnoticed. Definition regarding the time period since the initial trauma would define for how long the compression was necessary to induce metacarpal remodeling, but the presence of the foreign body and consequently, continuous stimulus of inflammation, would not permit a precise definition regarding the time period of the lesion, even if histopathology was authorized. Surgical removal of the rubber band associated with non-steroidal anti-inflammatory, analgesic, systemic and topical antibiotic (ointment) was considered satisfactory, leading to considerable improvement (normal gait) of the nociception and lameness at day three post-intervention and despite persistence of the bone radiographic aspect, full recovery of the skin lesion at day twenty-two.
\end{abstract}

Keywords: antibiotic, bone, osteomyelitis, radiology, trauma. 


\section{INTRODUCTION}

Despite its illusive inert appearance, bones demonstrate a high capability of adaptation. It is in constant rate of resorption and formation that are directly in dependence of mechanical stimulation. For example, it can be observed that at immobilized limbs bone resorption rate is increased, whilst in increased mechanical load, bone formation rate is higher [6].

Traumatic (e.g. automobile accidents) and pathologic (e.g. osteosarcoma) fractures, breed associated bone lesions (e.g. hip dysplasia, aseptic necrosis of the femoral head) are common diseases at the small animal routine and has its radiological and clinical aspects relatively well defined [14].

Differently, uncommon clinical cases generally produce unprecedented clinical signs and complementary exams patterns that could delay diagnosis and consequently, adequate treatment. That considered, clinical, laboratorial and radiographic aspects of a bone osteolysis caused by a compressive trauma by a rubber band is described.

\section{CASE}

It was attended at the Veterinary Hospital of the Dom Bosco Catholic University (UCDB), Campo Grande, MS, a 2-year-old intact female dog, mixed breed (long hair), with main complaint being an unresponsive to treatment lesion at the limb.

The patient had already being medicated systemic and topical antibiotic and anti-inflammatory treatment, with no response observed by the owner.

No previous history of cancer, other skin lesions, allergies, bug bites and any kind of inflicted trauma was reported.

At physical examination, it was observed lameness of the left thoracic limb and an ulcerative lesion was identified at its palmar surface (Figure 1A). Uncommonly, a $0.3 \mathrm{~cm}$ line shaped yellow object was identified at the center of the ulcer. Traction was made and the object distended $5 \mathrm{~cm}$ without breaking nor leaving the injury. The patient also showed signs of discomfort and at this point, with a foreign body (latex rubber band) as the main differential, general anesthesia was recommended for further diagnostic and treatment approach.

Complete blood count (CBC) and serum biochemistry (alanine aminotransferase, alkaline phosphatase, urea and creatinine) revealed only thrombocytopenia $(83.000 / \mu \mathrm{L})$ with no additional variations.
The patient was pre-medicated with acepromazine $^{1}(0.03 \mathrm{mg} / \mathrm{kg})$ and methadone $(0.2 \mathrm{mg} / \mathrm{kg})$ by intramuscular route. After $30 \mathrm{~min}$, a cephalic vein was punctured with a $22 \mathrm{G}$ catheter linked to a PRN lock. Anesthetic induction followed with propofol ${ }^{2}$ (4 mg/ $\mathrm{kg})$ and fentanyl ${ }^{3}(1 \mathrm{mcg} / \mathrm{kg})$ by intravenous route. After confirmation of absence of palpebral reflex (lateral and medial stimulation), the patient was positioned at right lateral recumbence for orotracheal intubation. Anesthetic maintenance was achieved with isoflurane ${ }^{2}$ diluted in $100 \%$ oxygen flow with $10 \mathrm{~mL} / \mathrm{kg}$ volume. With aid of an ultrasound and nerve localization device, the brachial plexus was blocked with levobupivacaine ${ }^{2}$ at $0.5 \%$ with a final volume of $0.5 \mathrm{~mL}$.

Under the long hair it was observed that the ulcerative lesion extended through all circumference of the limb (Figure 1B), although, at approximately median plane, the lesion transitioned for an erosion and further, a small scar.

Radiographic exam included dorsopalmar and mediolateral positioning (Figure 2A and B).

An exploratory surgery was performed for removal of the foreign body. Wide hair clipping was made, and wound cleaning protocol included cleaning with $1 \mathrm{~L}$ of sterile $0.9 \%$ saline solution ${ }^{4}$ followed by antisepsis with $2 \%$ and further $0.5 \%$ chlorhexidine 5 . A $3 \mathrm{~cm}$ incision was made following the way of the foreign body and with a small traction the object was removed (Figure 1C). Further cleaning proceeded followed by topical ointment ${ }^{1}$ (gentamycin sulfate $0.5 \mathrm{~g}$, sulfanilamide $5 \mathrm{~g}$, sulfadiazine $5 \mathrm{~g}$, urea $5 \mathrm{~g}$ and vitamin A 120.000 UI per $100 \mathrm{~g}$ ) and bandage.

Post-surgery prescription included clindamy$\operatorname{cin}^{6}(10 \mathrm{mg} / \mathrm{kg} /$ per os/every $12 \mathrm{~h} /$ during 10 days $)$, meloxicam ${ }^{7}(0.1 \mathrm{mg} / \mathrm{kg} /$ per os/every $24 \mathrm{~h} /$ during 3 days), dipyrone ${ }^{8}$ (25 mg/kg/per os/every $8 \mathrm{~h} /$ during 3 days) and topical ointment (previously described) every $12 \mathrm{~h}$, after topical cleaning with saline solution.

Twenty-two days post-surgery, at revaluation, it was observed only discrete improvement of bone remodeling of $\mathrm{V}$ metacarpal (Figure 2C) but with complete wound healing and full recovery of the lameness (Figure 2D).

\section{DISCUSSION}

Unfortunately, the reason that led to the presence of a latex compression band at the wrist of the patient was not clear. After discussion with the 


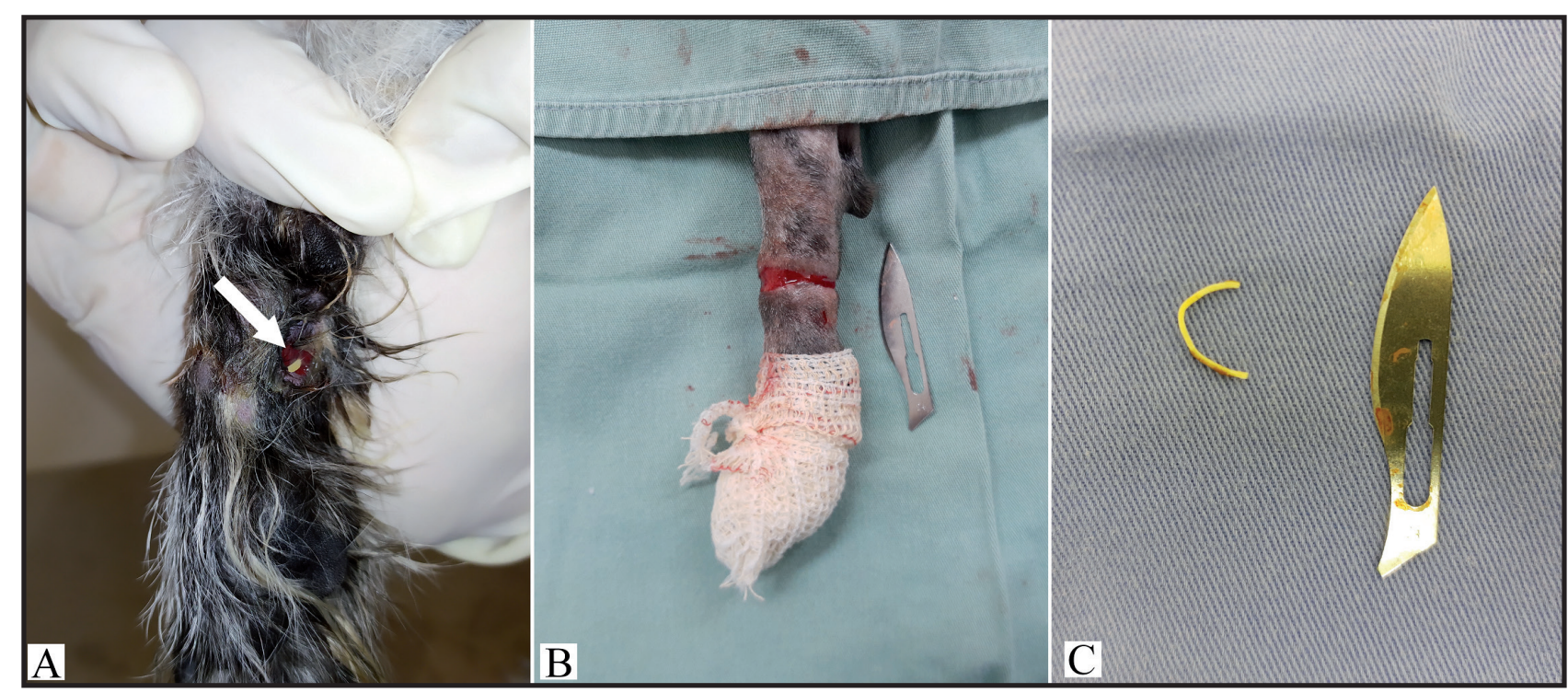

Figure 1. A- Ulcerative lesion at left palmar face in a canine patient, with a $0.3 \mathrm{~cm}$ line shaped yellow object at the center of the lesion (arrow). B- Lesion aspect after extensive hair clipping. C- Rubber band surgically removed from the inside of the lesion.

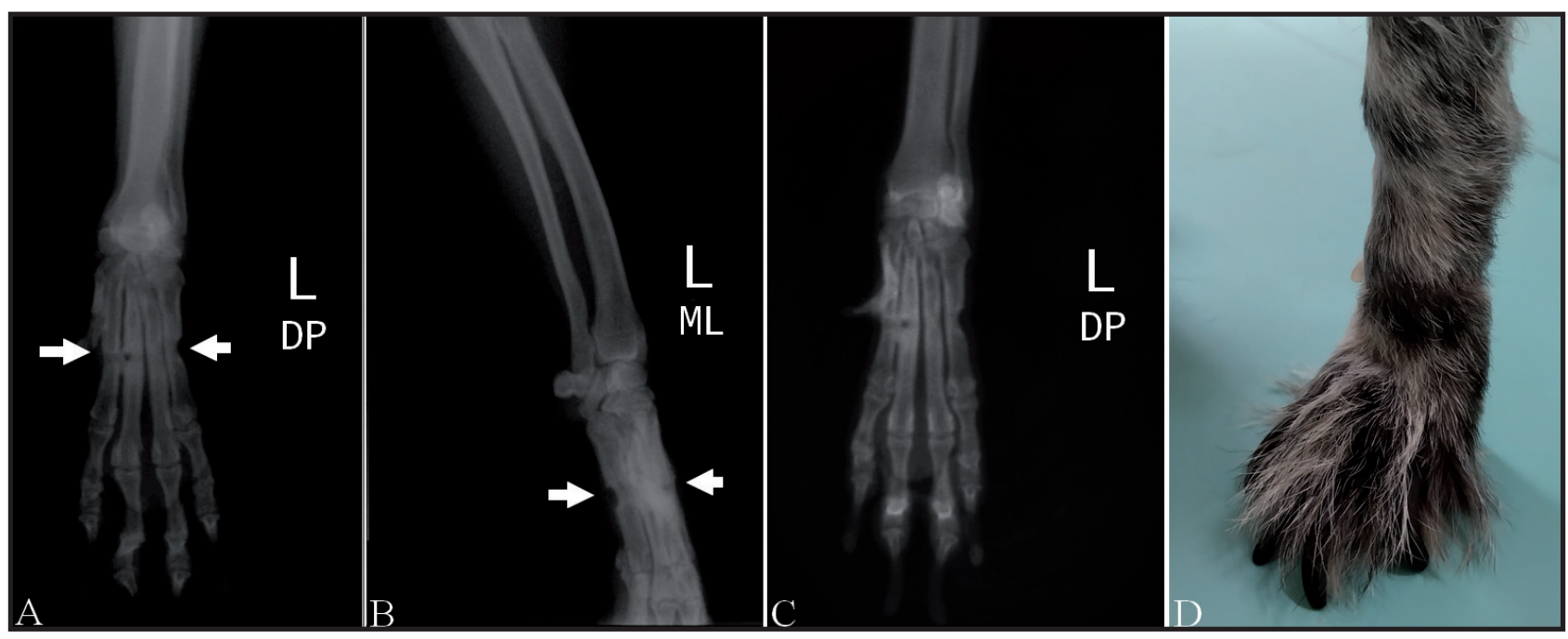

Figure 2. A \& B- Radiographic image of a canine patient metacarpals with increased radiopacity in bone tissue in the mid-diaphysis topography of the II, III, IV and V metacarpal bones, presence of bone remodeling with radiolucent area and slight bone loss (osteolysis) in the mid-diaphysis associated with discrete sclerosis of the medullary cavity of the II, III and V metacarpals. Dorsopalmar and mediolateral positioning, respectively. Lesion at the center of the arrows. C \& D- Same patient after 22 days post-treatment, radiographic (discrete improvement of bone remodeling of V metacarpal) and clinical aspect, respectively.

owner, the most probable cause was the possible use of a beauty accessory after a pet shop bathing service. Considering the patient's long hair, detachment of any accessory to a rubber band base could go unnoticed.

It is also unclear for how long the rubber band stayed in place to lead to such severe lesion. When questioned, the owner could only complain about a wound that would not heal for weeks. The estimative of time since the injury could be defined as well as 4 to 9 days, considering the presence of granulation tissue and signs of wound contraction $[3,9,12]$, but that could be misleading because the presence of the foreign body could constantly induce acute inflam- mation and alter the wound healing timeline, even if histopathology was authorized [1,9]. Nonetheless, the periosteal reaction could place the lesion between 7 to 14 days if the observed osteolysis was exclusive due to infection [11], but the compressive action of the rubber band was also in effect.

Although it may seem unlikely, the compression force was able to induce severe skin and bone lesions. The osteolysis pattern indicates that the compression could eventually lead to a complete transection of the metacarpals and potentially to amputation. Despite no literature was found reporting similar cases, tail docking in puppies (cosmetic and working dogs) 
and lambs (preventive for flystrike) are still a common practice in some countries [2,5,7] and may provide comparative information. The "rubber ring technique" for partial tail amputation is used for 3 to 5 days old puppies [7] and 7 days old lambs [2] and it takes up to 7 and 28 days, respectively, for complete transection of the tail. It should be considered that young animals still have immature bone structure and the rubber ring is recommended to be placed between coccygeal vertebrae, still, misplaced rings on the vertebrae body takes more time for transection, besides being more painful $[2,7]$. The physiopathology that leads to the tail amputation and the one that caused the lesions of the reported patient are potentially the same. The compressive stress of the rubber band creates ischemia which will eventually turn any posterior portion necrotic and detachment will be a consequence. At this case particularly, it is suspected that at first, skin and muscle lesion permitted the rubber band to penetrate tissue and be in direct contact with the metacarpals simultaneously to neovascularization of the skin above the rubber band, permitting blood flow to the distal phalangeal region, conserving the hand. Still, compression of the foreign body persisted at deep tissue and bones and led to the observed radiographic lesion, but, fortunately, at some point the rubber band broke and only foreign body inflammatory reaction took place.

The presence of thrombocytopenia alone, with no other CBC abnormalities, indicates that the persistent inflammation may have led to a depletion due to consumption. One of the most common causes of low platelet count may be for increased demand, such as it occurs in inflammation [13].

Pain management is also an important topic. Lameness in this case was not able to induce an important muscle atrophy, but that could be an aggravating to the patient considering the slow process of muscle strength and tonus recovery. Meloxicam and dypirone at the prescribed dosages showed satisfactory results, considering the owner report of considerable improvement of functional recovery of the limb from day 3 post-surgery.

Although the impossibility to perform a bacterial culture for a septic osteomyelitis diagnosis, if that the case, it was expected a neutrophilic leukocytosis $[4,8]$, which was not identified. Although it should be avoided empiric use of antibiotics, for suspected bacterial involvement at osteomyelitis (bone inflammation) and its potential to lead to severe clinical conditions, such as amputation [10], the authors considered imprudent leaving the patient without it. Prescription of clindamycin was considered necessary due to the extensive period of time of bone exposition through the foreign body.

The presence of the rubber band was a fortunate situation, considering that would be difficult to have certainty about the etiology responsible for the observed clinical alterations. Hopefully, the radiographic and clinical aspects described at this report may aid the diagnostic investigation of other professionals with similar cases.

\section{MANUFACTURERS}

${ }^{1}$ Vetnil Indústria e Comércio de Produtos Veterinários Ltda. Louveira, SP, Brazil.

${ }^{2}$ Cristália Produtos Químicos Farmacêuticos Ltda. Itapira, SP, Brazil.

${ }^{3}$ Janssen-Cilag Farmacêutica Ltda. São José dos Campos, SP, Brazil. ${ }^{4} J P$ Indústrica Farmacêutica S.A. Ribeirão Preto, SP, Brazil.

${ }^{5}$ Rioquímica Indústria Farmacêutica. São José do Rio Preto, SP, Brazil.

${ }^{6}$ Cepav Pharma LTDA. São Paulo, SP, Brazil.

${ }^{7}$ Ouro Fino Saúde Animal. Cravinhos, SP, Brazil.

${ }^{8}$ Farmace Indústria Químico-Farmacêutica Cearense Ltda. Barbalha, CE, Brazil.

Declaration of interest. The authors report no conflicts of interest. The authors alone are responsible for the content and writing of the paper.

\section{REFERENCES}

1 Abdulkhaleq L.A.,Assi M.A.,Abdullah R., Zamri-Saad M., Taufiq-Yap Y.H. \& Hezmee M.N.M. 2018. The crucial roles of inflammatory mediators in inflammation: A review. Veterinary World. 11: 627-635.

2 American Veterinary Medical Association (AVMA). 2014. Welfare Implications of Tail Docking of Lambs. Blackstone: AVMA, $4 \mathrm{p}$.

3 Balsa I.M. \& Culp W.T.N. 2015. Wound care. Veterinary Clinics Small Animal Practice. 45: 1049-1065.

4 Csébi P., Jakab C., Jánosi K., Sellyei B., Ipolyi T, Szabó Z., Arany-Tóth A. \& Németh T. 2010. Vertebral osteomyelitis and meningomyelitis caused by Pasteurella canis in a dog - Clinicopathological case report. Acta Veterinaria Hungarica. 58(4): 413-421. 
5 Diesel G., Pfeiffer D., Crispin S. \& Brodbelt D. 2010. Risk factors for tail injuries in Great Britain. Veterinary Record. 166: 812-817.

6 Dittmer K.E. \& Firth E.C. 2017. Mechanisms of bone response to injury. Journal of Veterinary Diagnostic Investigation. 29: 385-395.

7 Eyarefe O.D. \& Oguntoye C.O. 2016. Cosmetic tail docking: an overview of abuse and report of an interesting case. BMC Veterinary Research. 12(41): 1-4.

8 Farias M.R., Werner J., Ribeiro M.G., Rodigheri S.M., Cavalcante C.Z., Chi K.D., Condas L.A.Z., Gonoi T., Matsuzama T. \& Yazama K. 2012. Uncommon mandibular osteomyelitis in a cat caused by Nocardia africana. BMC Veterinary Research. 8: 239.

9 Hosgood G. 2006. Stages of Wound Healing and Their Clinical Relevance. Veterinary Clinics Small Animal Practice. 36: 667-685.

10 Kaya M., Okumus Z., Yanmaz L.E. \& Dogan E. 2011. Post-Traumatic osteomyelitis and its treatment in a dog. Pakistan Veterinary Journal. 31(4): 371-374.

11 Kealy J.K. \& McAllister H. 2005. Radiologia e Ultrassonografia do Cão e do Gato. 3.ed. Barueri: Manole, 436p.

12 Oliveira I.V.P.M. \& Dias R.V.C. 2012. Cicatrização de feridas: Fases e fatores de influência. Acta Veterinaria Brasilica. 6: 267-271.

13 Souza A.M., Pereira J.J., Campos S.D.E., Torres-Filho R.A., Xavier M.S., Bacellar D.T.L. \& Almosny N.R.P. 2016. Platelet indices in dogs with thrombocytopenia and dogs with normal platelet counts. Archivos de Medicina Veterinaria. 48: 277-281.

14 Thrall D.E. \& Robertson I.D. 2016. Atlas of Normal Radiographic Anatomy \& Anatomic Variants in the Dog and Cat. 2nd edn. Philadelphia: Elsevier, 319p. 